\title{
Formation of interfacial network structure via photo- crosslinking in carbon fiber/epoxy composites
}

\author{
S. H. Deng ${ }^{1}$, J. J. Zhao ${ }^{1}$, Q. F. Lin $^{2}$, C. J. Fan ${ }^{1}$, X. D. Zhou ${ }^{*}$ \\ ${ }^{1}$ State Key Laboratory of Chemical Engineering, East China University of Science and Technology, 130 Meilong Road, \\ 200237 Shanghai, PR China \\ ${ }^{2}$ School of Material Science and Engineering, East China University of Science and Technology, 130 Meilong Road, \\ 200237 Shanghai, PR China
}

\begin{abstract}
A series of diblock copolymers (poly(n-butylacrylate)-co-poly(2-hydroxyethyl acrylate))-b-poly(glycidyl methacrylate) ((PnBA-co-PHEA)- $b$-PGMA), containing a random copolymer block PnBA-co-PHEA, were successfully synthesized by atom transfer radical polymerization (ATRP). After being chemically grafted onto carbon fibers, the photosensitive methacrylic groups were introduced into the random copolymer, giving a series of copolymers (poly(n-butylacrylate)-co-poly(2-methacryloyloxyethyl acrylate))- $b$-poly(glycidyl methacrylate)((PnBA-co-PMEA)- $b$-PGMA). Dynamic mechanical analysis indicated that the random copolymer block after ultraviolet (UV) irradiation was a lightly crosslinked polymer and acted as an elastomer, forming a photo-crosslinked network structure at the interface of carbon fiber/epoxy composites. Microbond test showed that such an interfacial network structure greatly improved the cohesive strength and effectively controlled the deformation ability of the flexible interlayer. Furthermore, three kinds of interfacial network structures, i) physical crosslinking by H-bonds, ii) chemical crosslinking by photopolymerization, and iii) interpenetrating crosslinked network by photopolymerization and epoxy curing reaction were received in carbon fiber/epoxy composite, depending on the various preparation processes.
\end{abstract}

Keywords: polymer composites, random copolymers, interfacial network structure, photo-crosslinking

\section{Introduction}

Toughening of carbon fiber/epoxy composites has attracted a long-standing attention [1-4]. One of the most effective methods is by coating the carbon fibers with a ductile polymeric material to modify the mode of failure and thus the potential energy absorbing capacity of their composites [5-7]. However, many researches showed that thin coatings were finite in toughening the composites whereas thick coatings resulted in the reduction of composite strength and modulus $[8,9]$. In our previous study [10-13], similar results showing that the prolongation of the flexible blocks increased the thickness of the flexible layer and as a result the interfacial shear strength decreased due to the low cohesive strength and modulus of the flexible chains, were also obtained. Therefore, achievement of a ductile interlayer that has both high cohesive strength and large deformation capacity is one of the most effective methods to toughen the CF/epoxy composites without any significant loss in strength of the composite. There are various approaches to improve the strength and modulus of polymers. For instance, as we known, the strength of polymer is mainly reflected in the chemical bond of the main chain and the intermolecular force, and thus to increase the polarity of the polymer or generate hydrogen bond may improve the strength of the polymer. Secondly, the introduc-

\footnotetext{
${ }^{*}$ Corresponding author, e-mail: xdzhou@ecust.edu.cn

(C) BME-PT
} 
tion of the rigid polymer chain groups (such as phenyl, etc.) or increasing the degree of branching of the molecular chain will increase the strength of a polymer to some extent. In addition, the polymer chains with a suitable crosslinking can effectively increase the intermolecular forces, consequently avoiding the relative slippage of the molecular chains. As the degree of crosslinking increases, the deformation of the molecular chain will be reduced. Therefore, in this way, cohesive strength and deformation ability of polymers can be effectively controlled. Among the physical and chemical crosslinking methods [14, 15], photo-crosslinking exhibits considerable advantages, including mild reaction conditions, minimum byproduct formation, absence of potential toxic catalysts and initiators and easily controlled processing [16]. Consequently, in recent years there has been an increasing interest in utilizing photo-crosslinking as a means of biomaterial preparation in the field of medicinal and biomedical science [17-21]. However, to our knowledge, introduction of photosensitive materials into the interface between carbon fibers and resin matrix has never been reported.

In our previous study [13], we designed a series of diblock copolymers poly(n-butylacrylate)- $b$-poly (glycidyl methacrylate) (PnBA-b-PGMA) and introduced them into the interface between carbon fibers and epoxy resin. The assembly behavior of the grafted diblock copolymers at the interface was proved to be that the flexible PnBA block, having poor compatibility with the resin matrix, forms the interfacial flexible layer on the fiber surfaces, while the other block PGMA can react with the epoxy resin and stretch into the matrix. The introduction of the flexible layer could uniformly disperse the stress at interface, decrease the stress concentration, relax the residual stress and improve the toughness of the composites. However, when the PnBA block was too long, large deformation of the flexible layer would lead to easier interfacial slippage or damages due to the low cohesive strength and modulus of the PnBA block, resulting in the decreased interfacial shear strength.

Therefore, in this paper, we aim to improve the cohesive strength and modulus of the flexible chains and consequently control the deformation ability of the flexible interlayer through the formation of the interfacial network structure via photo-crosslinking. Following our previous consideration, we design and synthesize a diblock copolymer (poly(n-butylacrylate)-co-poly(2-hydroxyethyl acrylate) $)-b$ poly(glycidyl methacrylate) ((PnBA-co-PHEA)- $b$ PGMA) by atom transfer radical polymerization (ATRP). On one hand, the hydroxyl groups of the PHEA moieties in the flexible blocks can react with the functional groups on carbon fiber surfaces. On the other hand, the photo-crosslinkable groups (such as methacrylic groups, cinnamic groups, etc.) can be introduced into the flexible blocks by reacting with the hydroxyl groups. Additionally, by adjusting the molar ratio of nBA/HEA, the introduced amount of hydroxyl groups and thus the photo-crosslinkable groups can be controlled. With the introduction of such photo-crosslinkable groups into the interface, an interfacial network structure can be received under the ultraviolet (UV) irradiation. Afterwards, microbond test was employed to estimate the formation of the interfacial network structure in carbon fiber (CF)/epoxy resin composite.

\section{Experimental section}

\subsection{Materials}

The resin used in this study was the two part E51(618)/T-31 epoxy system, provided by Shanghai Resin Factory Co. Ltd. Carbon fibers (CFs) (T700$12 \mathrm{~K})$ were purchased from Toray Industries, Inc. $\mathrm{N}, \mathrm{N}, \mathrm{N}^{\prime}, \mathrm{N}^{\prime}, \mathrm{N}^{\prime \prime}$-pentamethyldiethylenetriamine (PMDETA, Aldrich, 99\%), $\alpha$-Bromoisobutyryl bromide (Aldrich, 98\%), 2-Hydroxyethyl acrylate (HEA, Aldrich, 96\%), and methacryloyl chloride (TCI Co., Ltd, $80 \%$ ) were used as received. n-Butyl acrylate (n-BA Shanghai Lingfeng Chemical Reagent Co., Ltd, AR grade) and glycidyl methacrylate (GMA Shanghai Yuanji Chemical Co., Ltd, AR grade) were purified by passing through a neutral aluminum oxide column, distilling under reduced pressure and then keeping in the presence of $4 \AA$ molecular sieve prior to use. $\mathrm{CuBr}$ (Sinopharm Chemical Reagent Co., Ltd, AR grade) and $\mathrm{CuCl}$ (Sinopharm Chemical Reagent Co., Ltd, AR grade) were purified by washing with glacial acetic acid, followed by absolute ethanol and ethyl ether and then dried under vacuum. Toluene (Aldrich, 99.8\%) and triethylamine (TEA, Aldrich, 99\%) were dried over calcium hydride $\left(\mathrm{CaH}_{2}\right)$ and then distilled under reduced pressure. Cyclohexanone (Shanghai No.1 Chemical Plant, AR grade) was also distilled under reduced pressure before use. 


\subsection{Polymerization procedures}

\subsubsection{Synthesis of diblock copolymer}

(PnBA-co-PHEA)-b-PGMA

The diblock copolymer (PnBA-co-PHEA)- $b$-PGMA was synthesized in two steps by atom transfer radical polymerization (ATRP), which depicted in Figure 1.

In a typical procedure, the random copolymer PnBAco-PHEA was first synthesized. The monomers of HEA and nBA dissolved in cyclohexanone were charged in a three-neck round-bottom flask $(250 \mathrm{~mL})$, followed by adding the catalyst $\mathrm{CuBr}$ and ligand PMDETA with a ratio of 1:1. After being degassed and back-filled with nitrogen three times, the solution became a stable complex under a continuous stirring. Then the initiator EBriB was injected in it via a syringe, simultaneously immersed in a thermostated water bath of $70^{\circ} \mathrm{C}$ to initiate the polymerization. When the polymerization was stopped, the polymer solution was diluted with THF, passed through a column of neutral alumina to remove $\mathrm{Cu}-$ catalyst. After concentrated and precipitated twice in ether, and once more in methanol/water (1:1), the random copolymer Br-terminated PnBA-co-PHEA was dried under vacuum and then used as macroinitiator to initiate the polymerization of diblock copolymers.

Second, the diblock copolymer (PnBA-co-PHEA)$b$-PGMA was synthesized in a similar manner to the polymerization of PnBA-co-PHEA, except Br-terminated PnBA-co-PHEA was used as the macroinitiator, $\mathrm{CuCl}$ as the catalyst, GMA as the monomer and $\mathrm{n}$-hexane as precipitator. After the polymerization was conducted in a $30^{\circ} \mathrm{C}$ water bath for $3 \mathrm{~h}$, the diblock copolymer was purified in a similar way to that described for the random copolymer Br-terminated PnBA-co-PHEA.

\subsubsection{Synthesis of copolymer PnBA-co-PMEA}

To study the ability of the copolymer PnBA-coPHEA to introduce photosensitive groups and subsequent photopolymerization upon UV irradiation, copolymers poly(n-butylacrylate)-co-poly(2-methacryloyloxyethyl acrylate) (PnBA-co-PMEA) were prepared as follows: first, the as-prepared copolymers Br-terminated PnBA-co-PHEA, dissolved in toluene, and excess anhydrous TEA were charged into a $500 \mathrm{~mL}$, three-necked, round-bottom flask, which was cooled at $0^{\circ} \mathrm{C}$ in ice water. Then methacryloyl chloride, diluted with dry toluene, was added dropwise under a nitrogen environment. The reaction mixture was kept stirring at room temperature for 2 days, followed by filtering carefully to remove triethylamine hydrochloride salt. After being concentrated and precipitated twice in n-hexane, the endproduct was finally dried under vacuum overnight at $40^{\circ} \mathrm{C}$. The synthetic illustration of PnBA-coPMEA was shown in Figure 1.

\subsection{Treatment of carbon fibers}

\subsubsection{Pre-treatment of carbon fibers}

The carbon fibers as received were first extracted with acetone and petroleum ether in a Soxhlet apparatus for $24 \mathrm{~h}$ respectively, and then dried under vacuum at $60^{\circ} \mathrm{C}$, denoted as $\mathrm{CF} 0$. Then, the chemical oxidation of $\mathrm{CF} 0$ was performed by refluxing in nitric acid $\left(\mathrm{HNO}_{3}\right)$ for $5 \mathrm{~h}$ at $100^{\circ} \mathrm{C}$. The end-product was finally washed with distilled water to neutral $\mathrm{PH}$, and dried under vacuum overnight at $60^{\circ} \mathrm{C}$, named CF-COOH.
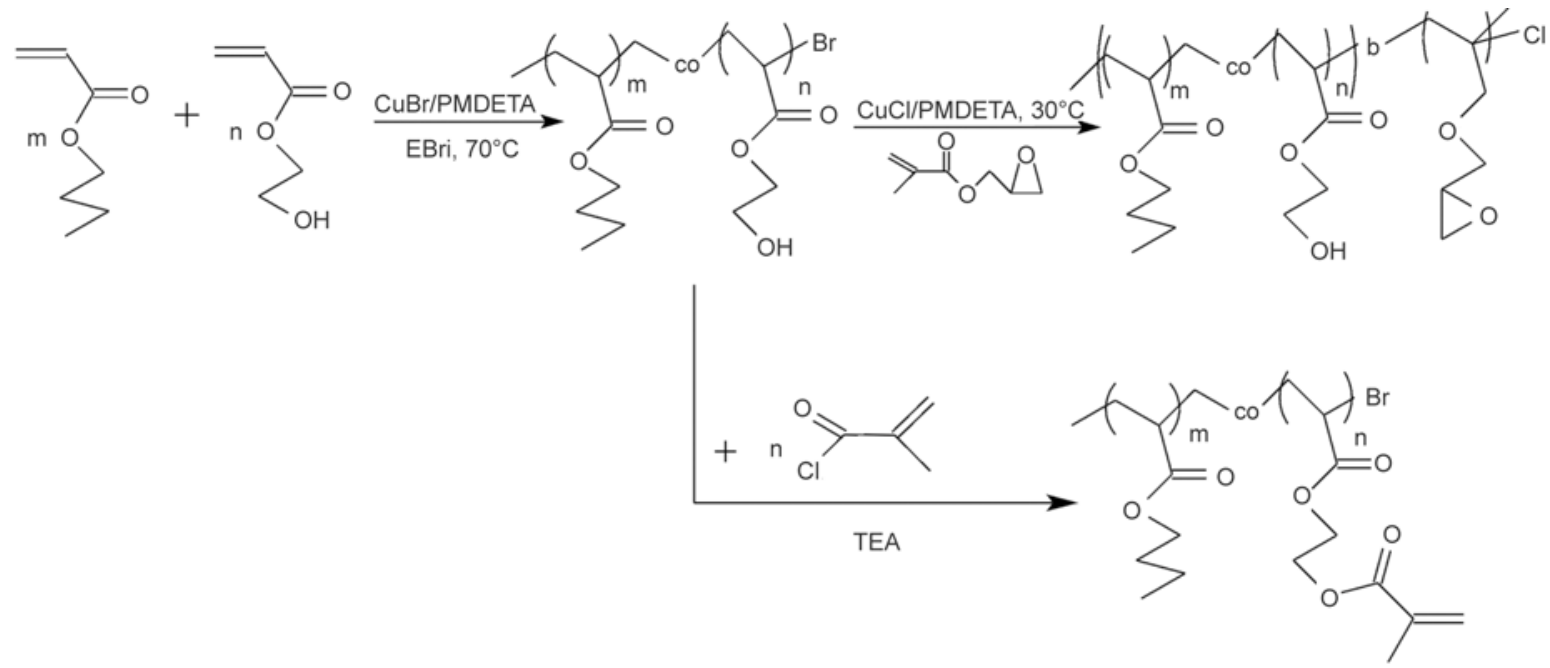

Figure 1. Synthetic routes of the copolymers (PnBA-co-PHEA)- $b$-PGMA and PnBA-co-PMEA 


\subsubsection{Grafting of diblock copolymers onto CFs}

The as-prepared CF-COOH was immersed in thionyl chloride and stirring at $70^{\circ} \mathrm{C}$ for $24 \mathrm{~h}$. The residual thionyl chloride was removed by reduced pressure distillation, giving acyl chloride-functionalized CF$\mathrm{COCl}$. Then, $2.4 \mathrm{~g}$ (PnBA-co-PHEA)- $b$-PGMA dissolved in $120 \mathrm{~mL}$ anhydrous dioxide was added immediately. The reactor was degassed under vacuum and back-filled with pure nitrogen for three times, and elevated temperature to $90^{\circ} \mathrm{C}$. Finally, CFs grafted with diblock copolymer (PnBA-coPHEA )- $b$-PGMA were taken out after $48 \mathrm{~h}$, and extracted with acetone in a Soxhlet apparatus for $48 \mathrm{~h}$ to remove the physisorbed copolymers. After dried under vacuum overnight at $40^{\circ} \mathrm{C}$, the grafted endproduct was denoted as CF-PHEA. The typical grafting procedure was described as Figure 2.

\subsubsection{Methacrylic functionalization of copolymers}

A dried round bottom made-to-order reactor equipped with stirrer and pressure equalizing addition funnel was charged with $0.5 \mathrm{~g} \mathrm{CF}$-(PnBA-co-PHEA)- $b$ PGMA and $150 \mathrm{~mL}$ of dry toluene. After stirring for 15 min to mix uniformly under nitrogen atmosphere, $1.2 \mathrm{~mL}$ of triethyl amine were added in under continuous stirring. Subsequently, $0.5 \mathrm{~mL}$ methacryloyl chloride, diluted with $25 \mathrm{~mL}$ of dry toluene, were added dropwise to the reactor. After reacting at room temperature for 2 days under nitrogen atmosphere and continuous stirring, the CF-(PnBA-co-PMEA)$b$-PGMA was obtained and named CF-PMEA. The schematic illustration of the preparation of CF-
PMEA was shown in Figure 2. After that, the samples of CF-PMEA were extracted with THF in a Soxhlet apparatus for $24 \mathrm{~h}$, and then dried under vacuum overnight at $40^{\circ} \mathrm{C}$ before use.

\subsubsection{UV irradiation}

The random copolymers PnBA-co-PMEA were first dissolved in THF and uniformly coated on glass slide. After solvent evaporation, the films of the random copolymers adhered to glass slide, were exposed to a $1000 \mathrm{~W}$ high-pressure mercury lamp (LT-101, Blue Sky UV and IR Techmology Development Co.) with a distance of around $10 \mathrm{~cm}$ for a certain time. The carbon fibers grafted with diblock copolymers (PnBA-co-PMEA)- $b$-PGMA were divided into two groups to subject to UV irradiation. One was first incorporated with epoxy resin and cured, then subjected to UV irradiation, denoted as curing-crosslinking samples; the other was first subjected to UV irradiation and then incorporated with epoxy resin and cured, named as crosslinking-curing samples. The samples were subjected to UV irradiation in a similar manner with that of the random copolymers.

\subsection{Preparation of the microbond testing samples}

The detail of the preparation of CF/epoxy micro-composite for microbond test was same as that used in our previous study [22].

\subsection{Instrument and measurements}

Fourier transform infrared (FTIR) spectra of samples were recorded on a Thermo Electron Corporation

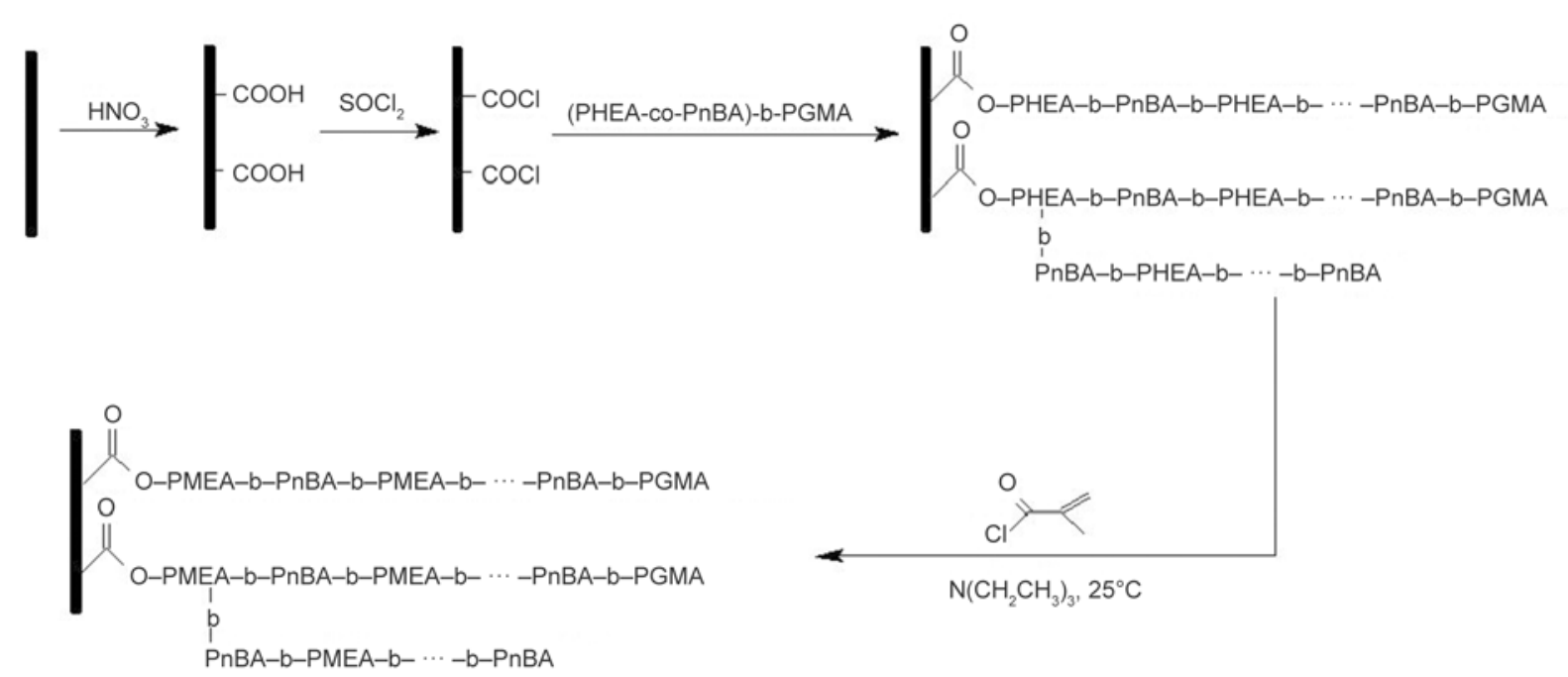

Figure 2. Schematic illustration of grafting block copolymer onto $\mathrm{CF}$ and subsequent methacrylic functionalization of the grafted copolymers 
Nicolet 5700. The block copolymers were ground with potassium bromide $(\mathrm{KBr})$ and pressed into pieces before FTIR test. CFs grafted with copolymers was recorded by attenuated total reflection of Fourier transform infrared spectroscopy (ATRFTIR) using Ge crystal plate and Smart iTR accessory.

${ }^{1} \mathrm{H}-\mathrm{NMR}$ spectra were recorded in deuterated acetone using a Bruker DSX $300 \mathrm{MHz}$ spectrometer with the typical acquisition parameters.

A twice-detector gel permeation chromatography (GPC), equipped with an refractive index detector (Wyatt Technology Corp., Optilab REX) and a multiangle static laser light scattering detector (Wyatt Technology Corp., DAWN HELEOS), was used to determine the absolute molecular weight and molecular weight distribution of diblock copolymers. The GPC was operated at a normal flow rate of $1 \mathrm{~mL} / \mathrm{min}$ at $45^{\circ} \mathrm{C}$, DMF with $0.05 \mathrm{M} \mathrm{LiBr}$ used as eluent.

Scanning electron microscopy (SEM) was applicable to investigate the surface morphology of the carbon fibers. Samples were fixed to a platform with conductive tape, vacuum-dried and sputtered with gold. Images were taken on a HITACHI S-4800 scanning electron microscopy, operating at $15 \mathrm{kV}$. A TA DMAQ800 dynamic mechanical thermal analyzer (USA) was employed to test the glass transition temperature of samples. The random copolymers were loaded into the lower tray of powder clamp used in conjunction with a dual cantilever clamp, and then placing the upper cover plate on top of the sample. After the filled clamp was installed, the test was performed from -60 to $80^{\circ} \mathrm{C}$ at $3^{\circ} \mathrm{C} / \mathrm{min}$ with constant oscillation amplitude of $20 \mu \mathrm{m}$ and frequency of $1 \mathrm{~Hz}$.

The microbond test was performed on a YG004A Electronic single fiber tensile strength tester, provided by Changzhou No.2 Textile Machinery Co. Ltd. The interfacial shear strength (IFSS) was calculated as in the literature [23] and more than 30 specimens were successfully tested for each composite.

\section{Results and discussion}

\subsection{Structure characterization of copolymers}

Figure 3 shows the FTIR spectra of the obtained copolymers PnBA-co-PHEA and (PnBA-co-PHEA)$b$-PGMA. The absorption peaks at 2960 and $2870 \mathrm{~cm}^{-1}$ represent the alkane $\mathrm{C}-\mathrm{H}$ stretching vibra-

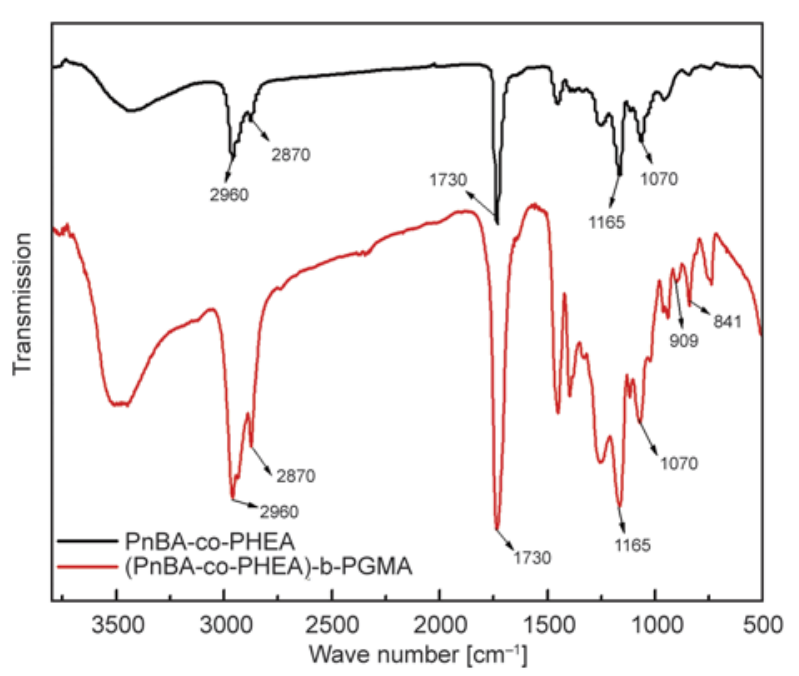

Figure 3. FTIR spectra of copolymers PnBA-co-PHEA and (PnBA-co-PHEA)- $b$-PGMA

tions. The characteristic peak at $3430 \mathrm{~cm}^{-1}$ is assigned to the characteristic absorption of $-\mathrm{OH}$ group. The strong characteristic peak at $1730 \mathrm{~cm}^{-1}$ is attributed to the $\mathrm{C}=\mathrm{O}$ stretching vibration. The absorption peaks at 1165 and $1070 \mathrm{~cm}^{-1}$ correspond to the characteristic absorption of $\mathrm{C}-\mathrm{O}$ group. These characteristic absorption peaks suggested that the monomer nBA and HEA were involved in the ATRP copolymerization. Furthermore, comparing with PnBA-co-PHEA, the FTIR spectrum of (PnBA-coPHEA)- $b$-PGMA exhibited the characteristic peak of the epoxy ring at 909 and $841 \mathrm{~cm}^{-1}$, indicating the successful synthesis of the block copolymer (PnBA$c o$-PHEA)- $b$-PGMA by ATRP.

The structure of the copolymer PnBA-co-PHEA was also characterized by ${ }^{1} \mathrm{H}-\mathrm{NMR}$. Based on the composition of copolymers determined by ${ }^{1} \mathrm{H}-\mathrm{NMR}$, the reactivity ratio of $\mathrm{HEA} / \mathrm{nBA}$ monomer pair is estimated to be $r_{\mathrm{HEA}}=1.505$ and $r_{\mathrm{nBA}}=0.894$ by KelenTüdős method, which has been reported in our previous paper [24], suggesting that the PnBA-coPHEA was random copolymers. Therefore, there is a uniform random distribution of hydroxyl groups in PnBA-co-PHEA and thus the photosensitive groups in copolymer PnBA-co-PMEA after the esterification of hydroxyl groups with methacryloyl chloride, which may reduce the phenomenon that the local crosslinking density is too large during the formation of the interfacial crosslinking network.

The absolute molecular weights and molecular weight distributions of the synthesized copolymers were determined by GPC. The RI traces of GPC of PnBA-co-PHEA and (PnBA-co-PHEA)- $b$-PGMA 
Table 1. Molecular weights, distributions and molar ratio of nBA/HEA of the synthesized random copolymers PnBA-coPHEA

\begin{tabular}{|c|c|c|c|c|}
\hline Random copolymers & $\begin{array}{c}\overline{\mathbf{M}}_{\mathbf{n}, \mathbf{G P C}} \\
{[\mathbf{g} / \mathbf{m o l}]}\end{array}$ & $\begin{array}{c}\overline{\mathbf{M}}_{\mathbf{w}, \mathbf{G P C}} \\
{[\mathbf{g} / \mathbf{m o l}]}\end{array}$ & $\overline{\mathbf{M}}_{\mathbf{w}} \overline{\mathbf{M}}_{\mathbf{n}}$ & $\begin{array}{c}\text { Molar ratio of } \\
\mathbf{n B A} / \mathbf{H E A}\end{array}$ \\
\hline${\text { (PnBA-co-PHEA })_{190}}$ & 23400 & 31920 & 1.36 & $160: 30$ \\
\hline${\text { (PnBA-co-PHEA })_{220}}_{(\text {PnBA-co-PHEA })_{300}}$ & 28470 & 33940 & 1.19 & $160: 60$ \\
\hline${\text { (PnBA-co-PHEA })_{310}}$ & 37410 & 53600 & 1.43 & $260: 40$ \\
\hline (PnBA-co-PHEA $)_{320}$ & 40910 & 53760 & 1.31 & $210: 100$ \\
\hline
\end{tabular}

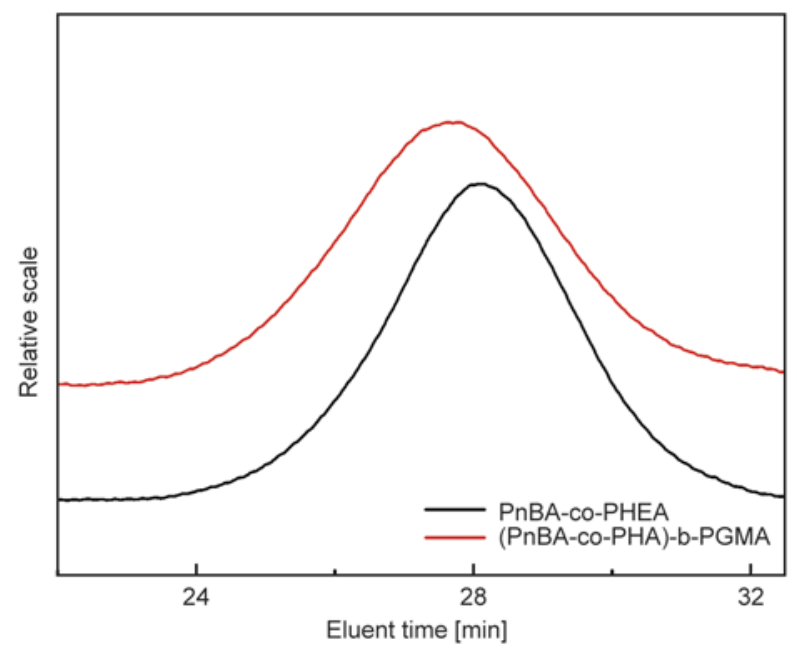

Figure 4. The RI traces of GPC of copolymers PnBA-coPHEA and (PnBA-co-PHEA)- $b$-PGMA

were presented in Figure 4, and the calculated values for random copolymers PnBA-co-PHEA were listed in Table 1. It can be seen that both GPC traces of the random copolymer and block copolymer in Figure 4 are unimodal, and their molecular weight distributions is relatively narrow $\left(\bar{M}_{\mathrm{w}} / \bar{M}_{\mathrm{n}}\right)$, as seen in Table 1. A series of random copolymers of various polymerization degrees and molar ratio of $\mathrm{nBA} /$ HEA were successfully synthesized. Besides, the eluent time for block copolymer is smaller than that of the random copolymer, and the peak of the block copolymer is shifted toward higher molecular weight position in Figure 4, indicating that the random copolymers can further initiate the polymerization of GMA monomers to prepare some kinds of block copolymers. Furthermore, the polymerization degree of GMA can be calculated from the absolute molecular weight and is about 100 110 for all the diblock copolymers.

\subsection{Surface analysis of carbon fibers}

The ATR-FTIR spectra of CF0 and CF-PHEA are shown in Figure 5. Compared to $\mathrm{CF} 0$, some new peaks of ester $\left(-\mathrm{C}=\mathrm{O}, 1730 \mathrm{~cm}^{-1}\right)$, ether $(-\mathrm{C}-\mathrm{O}-\mathrm{C}$, 1165 and $1070 \mathrm{~cm}^{-1}$ ) and epoxy ring (909 and

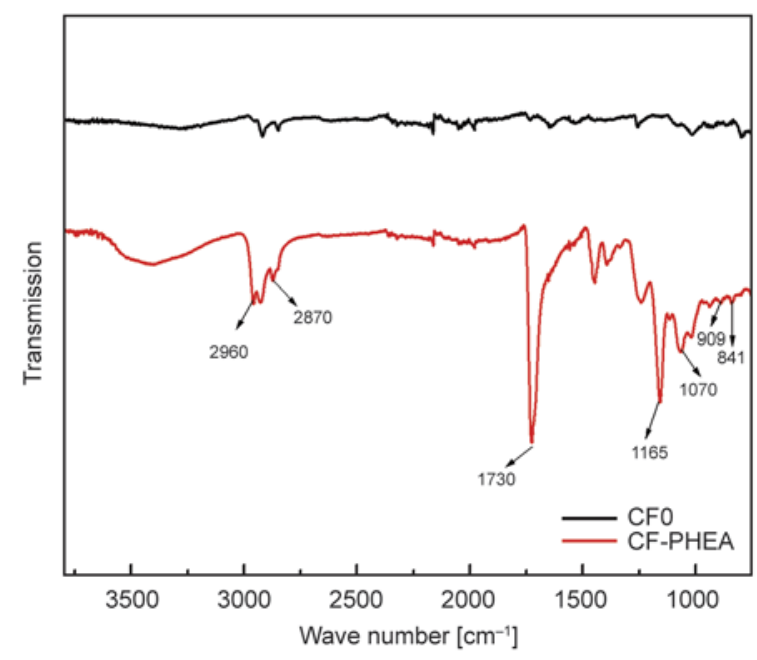

Figure 5. ATR-FTIR spectra of CF0 and CF-PHEA

$841 \mathrm{~cm}^{-1}$ ) that are all the characteristics of pure (PnBA-co-PHEA)-b-PGMA (Figure 3) appeared in ATR-FTIR spectra of CF-PHEA, indicating that (PnBA-co-PHEA)-b-PGMA chains have been successfully grafted onto CF surfaces.

It can be also seen from SEM micrographs of CFs (see Figure 6) that, the CF0 desized the commercial coatings has a relatively smooth surface (Figure 6a), whereas for the grafted $\mathrm{CF}$, there are a few polymers deposited on the fiber substrate, resulting in a rough surface as shown in Figure 6b. Since the CFs were thoroughly washed by acetone to remove the physisorbed polymers after the grafting reaction, it would be reliable to conclude that the block copolymer (PnBA-co-PHEA)-b-PGMA was successfully chemically-grafted onto carbon fiber surface.

\subsection{Photo-crosslinking of random copolymer PnBA-co-PMEA}

To study the ability of the random copolymer PnBA-co-PHEA to introduce photosensitive groups and subsequent photopolymerization upon UV irradiation, the copolymer PnBA-co-PMEA was synthesized and initiated photopolymerization. Figure 7 shows the ${ }^{1} \mathrm{H}-\mathrm{NMR}$ and ATR-FTIR spectra of the random copolymer PnBA-co-PMEA. 


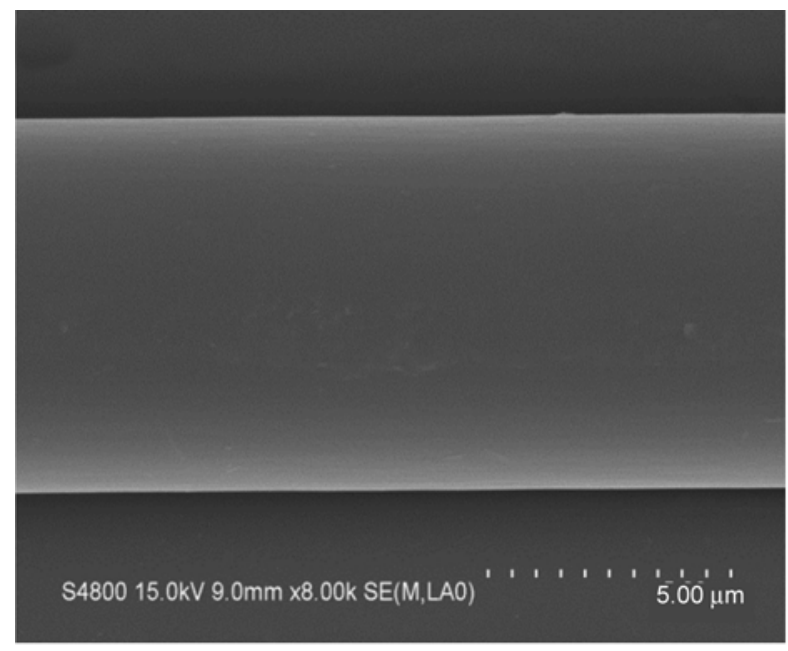

a)

Figure 6. SEM micrographs of CF0 (a) and CF-PHEA (b)

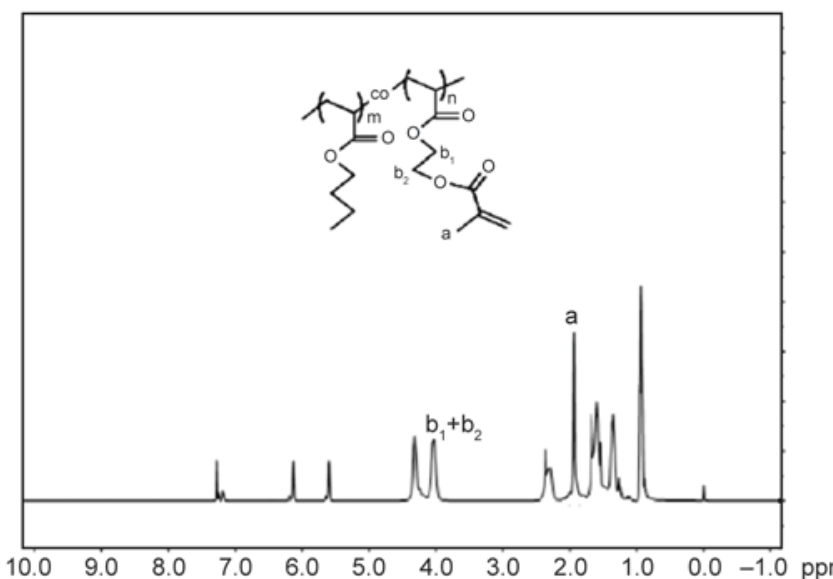

b)

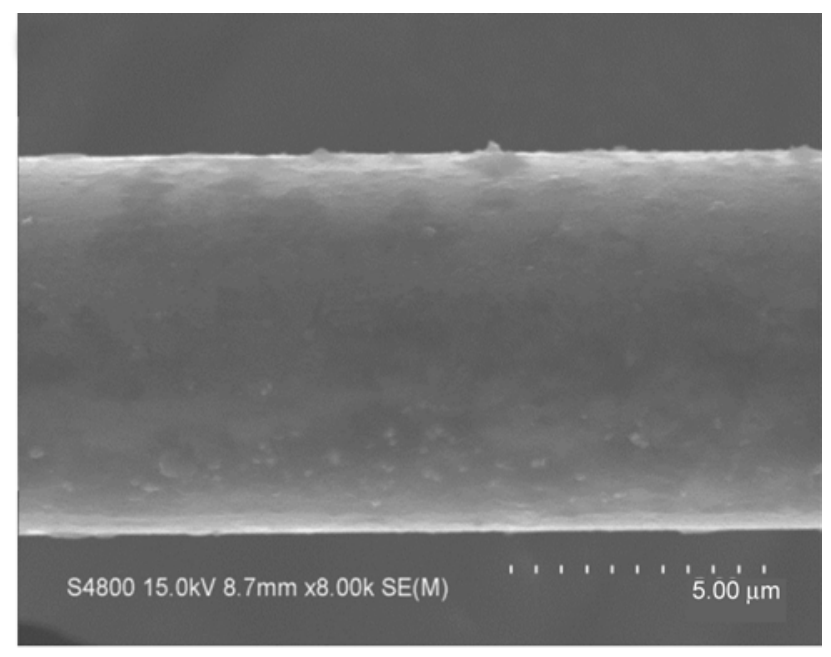

b)

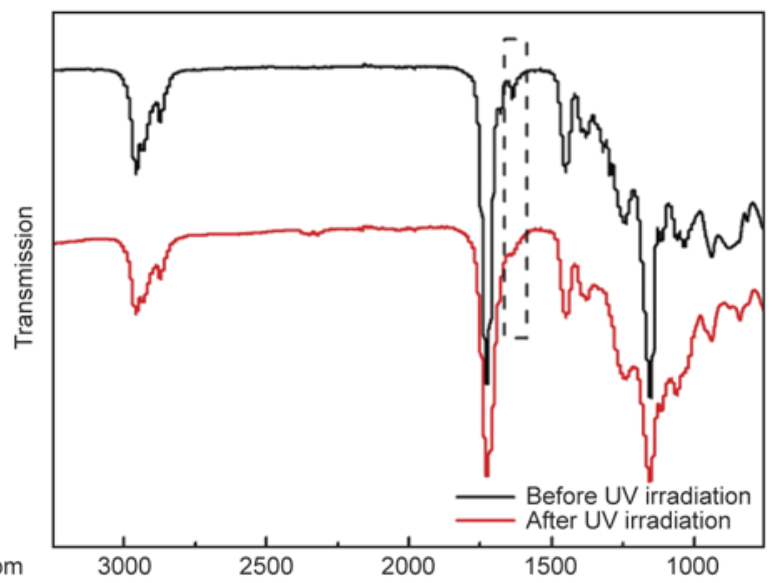

a)

Wave number $\left[\mathrm{cm}^{-1}\right]$

Figure 7. (a) ${ }^{1} \mathrm{H}-\mathrm{NMR}$ spectrum of random copolymer $\mathrm{PnBA}_{160}-\mathrm{co}-\mathrm{PMEA}_{40}$; (b) ATR-FTIR spectra of copolymers PnBAco-PMEA before and after UV irradiation

It can be found from Figure 7a that the peak of the methylene protons adjacent to the hydroxyl groups in random copolymer PnBA-co-PHEA at $3.8 \mathrm{ppm}$ [24] completely disappeared. The peak (a) at $1.9 \mathrm{ppm}$ is assigned to the methyl protons in methacrylic (MA) groups, while the peak $\left(b_{1}+b_{2}\right)$ at $4.0 \mathrm{ppm}$ is due to the methylene protons adjacent to the MA groups. From the intensity of the two isolated and sharp peaks, we could accurately calculate their molar ratio to be $3 / 3.96$, which is extremely close to that when the hydroxyl groups in PHEA moieties are totally substituted by the MA groups (3/4). Thus, it can be concluded that methacrylic functionalization of the random copolymer PnBA-co-PHEA, giving PnBA-co-PMEA, was completed under the reactive condition in this study.

The ATR-FTIR spectra of random copolymers PnBA-co-PMEA before and after UV irradiation are shown in Figure 7b. Compared with the FTIR spectrum of PnBA-co-PHEA (see Figure 3), the characteristic peak at $1636 \mathrm{~cm}^{-1}$ attributed to stretching vibration of the double bonds in methacrylic groups was observed, indicating the successful esterification of methacryloyl chloride with hydroxyl groups of the random copolymer PnBA-co-PHEA. After UV irradiation, the infrared absorption intensity at $1636 \mathrm{~cm}^{-1}$ of methacrylic double bonds in copolymer PnBA-co-PMEA decreased. This is attributed to the photopolymerizationof the methacrylic double bonds upon UV exposure, reported by many researchers [25-28] and schematically shown in Figure 8. Consequently, the content of methacrylic double bonds in PnBA-co-PMEA copolymers significantly reduced, corresponding to the decreased absorption intensity at $1636 \mathrm{~cm}^{-1}$ as illustrated in Figure $7 b$. 


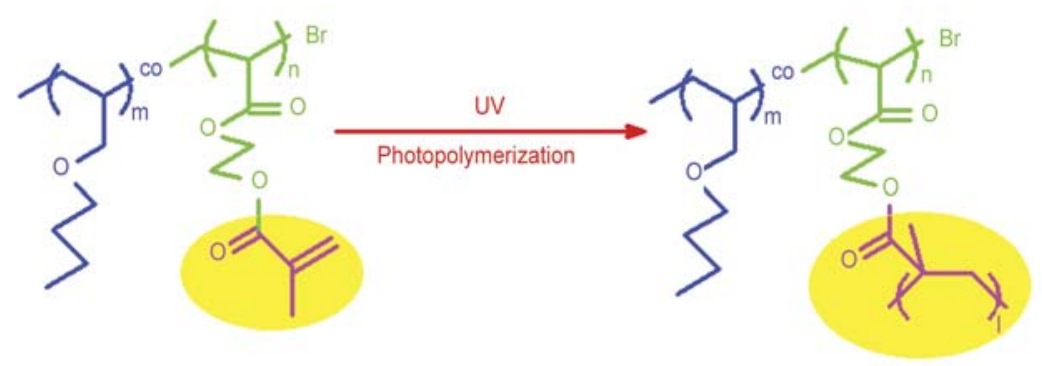

Figure 8. Schematic illustration of photopolymerization of copolymers PnBA-co-PMEA

The glass transition temperatures $\left(T_{\mathrm{g}}\right)$ of the copolymers PnBA160-co-PHEA 160 and PnBA160-coPMEA $_{160}$ were evaluated by DMA and shown in Figure 9. It was found that all of the copolymers exhibited a single glass transition peak, conforming to the characteristic of the random copolymers. Compared to PnBA-co-PHEA, PnBA-co-PMEA that introduces MA groups, had no obvious increase in the glass transition temperature, only shifting from -27.7 to $-26.2^{\circ} \mathrm{C}$. This suggested that the photocrosslinkable copolymer PnBA-co-PMEA retained the good flexibility of the random copolymer block. However, after UV irradiation, the transition loss peak decreased in intensity, broadened, and shifted to higher temperature of $-16.5^{\circ} \mathrm{C}$, which is the characteristic of crosslinking reaction and indicates the formation of the crosslinked network structure by the photopolymerization reaction [29]. It is noted that the glass transition temperature is still below zero temperatures, implying that the random copoly-

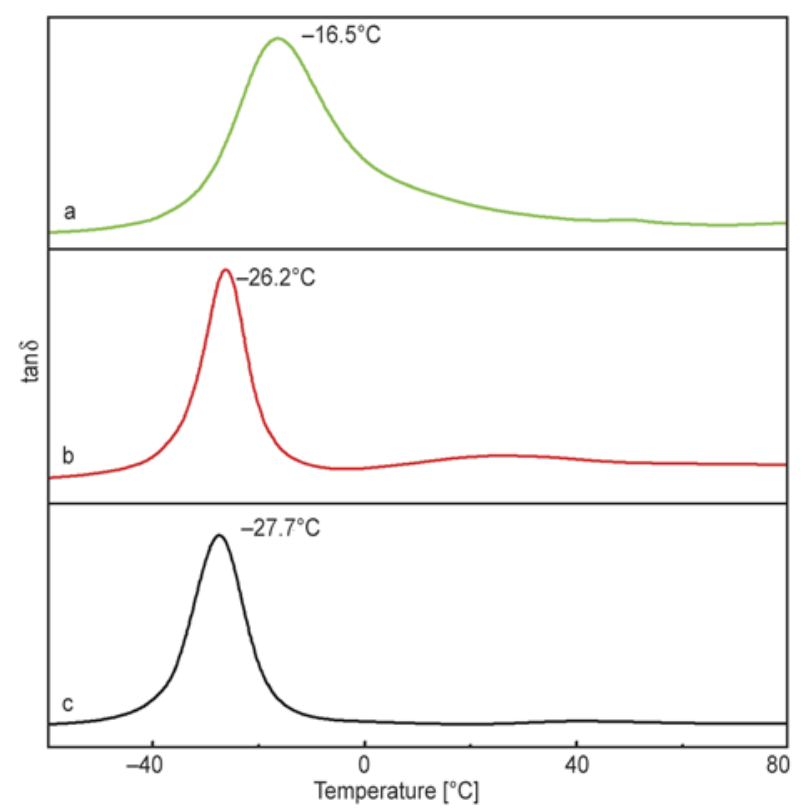

Figure 9. DMA curves of various copolymer samples (a) PnBA-co-PHEA; (b) PnBA-co-PMEA without UV irradiation; and (c) PnBA-co-PMEA after UV irradiation for $30 \mathrm{~min}$ mer block after UV irradiation is a lightly crosslinked polymer and acts as an elastomer.

\subsection{Formation of interfacial network structure}

\subsubsection{Physical crosslinking by $\mathbf{H}-$ bonds}

Undoubtedly, the interfacial molecular structure can significantly determine the mechanical properties of interface region such as strength and modulus etc. and thus the bonding of fiber-interface region and interface region-matrix. Therefore, interfacial adhesion strength may be, in some cases, a response to interfacial molecular structure as matrix and fiber are fixed. In this paper, we first measured the interfacial shear strength of CF-PHEA/epoxy and CFPMEA/epoxy micro-composites, respectively to investigate the variation of the interfacial molecular structure.

Figure 10 showed the IFSS results of epoxy resin micro-composites reinforced with different CFs. Due to the increased oxygen-containing functional groups on CF surface by oxidation, the IFSS value of CF-COOH composite increase d from $29.8 \pm 2.8$

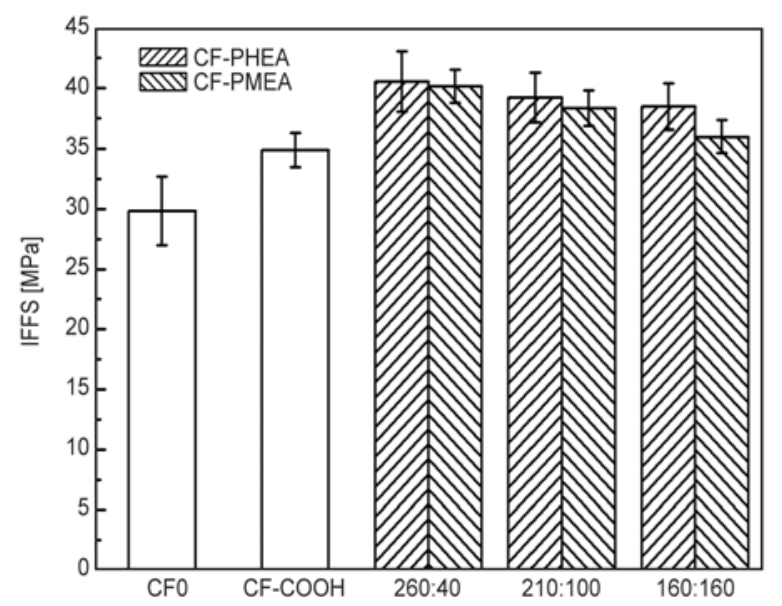

Figure 10. Influence of surface modification on the interfacial shear strength of CF/epoxy micro-composites $(260: 40,210: 100$ and 160:160 are the molar ratio of $\mathrm{nBA} / \mathrm{HEA}$ or $\mathrm{nBA} / \mathrm{MEA}$ in the grafted copolymers with the same length of PGMA) 


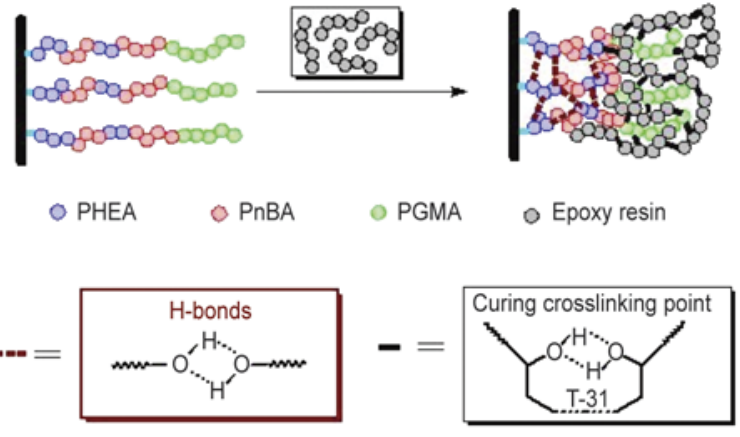

Figure 11. Illustration of the formation of the interfacial network structure crosslinking by $\mathrm{H}$-bonds

to $34.9 \pm 1.4 \mathrm{MPa}$, in consistent with the results of our previous study [13]. Furthermore, with the introduction of diblock copolymers (PnBA-co-PHEA)- $b$ PGMA or (PnBA-co-PMEA)- $b$-PGMA into the interface, the interfacial adhesion of the composites was further improved, partly resulting from that the PGMA blocks in diblock copolymers can be involved in the curing reaction of the epoxy matrix. On the other hand, the existence of the interfacial flexible layer, forming by the other blocks PnBA-co-PHEA or PnBA-co-PMEA, can effectively transfer loads through its deformation and chain-movement, contributing to the increased IFSS values in CF-PHEA and CF-PMEA composites.

It was noted that all the IFSS values of CF-PHEA/ epoxy composite are higher than those of the corresponding CF-PMEA/epoxy composite as illustrated in Figure 10. For CF-PHEA/epoxy composite, since the PHEA moieties in the grafted diblock copolymer contain many hydroxyl groups, it is easy to form the H-bonds between the PHEA moieties in the flexible layer (see Figure 11) [30]. It can be also reflected from the result in Figure 10 that the $T_{\mathrm{g}}$ of PnBA-co-PMEA is only slightly higher than that of PnBA-co-PHEA. It is supposed to be that the substitution of MA groups (methacrylic double bonds) for hydroxyl groups can strongly increase the $T_{\mathrm{g}}$ of PnBA-co-PMEA, but this effect is partly offset by the destroy of the $\mathrm{H}$-bonds due to the disappearance of hydroxyl groups. However, in the CF-PMEA/ epoxy composite, the interfacial structure without crosslinking possesses low cohesive strength and modulus, although PnBA-co-PMEA has higher $T_{\mathrm{g}}$ than that of PnBA-co-PHEA. Thus, the formation of the interfacial network structure in CF-PHEA/ epoxy composite, crosslinking by H-bonds, improved the strength and modulus of the flexible layer to a certain extent, and thereby the interfacial shear strength.

Furthermore, we can see that with an increase in the molar units of PHEA in the random copolymer PnBA-co-PHEA from 40 to 100 and 160, the IFSS value had a slight decrease. This indicates that the hydroxyl groups in PHEA moieties prefer to forming the $\mathrm{H}$-bonds between each other, rather than being involved in the curing reaction of the epoxy resin in the presence of the PGMA blocks, because if the hydroxyl groups tend to involve in the curing reaction, the increase in the molar units of PHEA will be beneficial to the improvement in the interfacial adhesion. The slight decrease in IFSS resulted from that the total length of the flexible chain increased from $300(\mathrm{PnBA} / \mathrm{PHEA}=260: 40)$ to 310 $(\mathrm{PnBA} / \mathrm{PHEA}=210: 100)$ and $320(\mathrm{PnBA} / \mathrm{PHEA}=$ 160:160). A more significant trend to decline was observed in the CF-PMEA/epoxy composite due to the destruction of the physical crosslinking by $\mathrm{H}$ bonds. The increase in the total length of the flexible chain resulted in the larger deformation and interfacial slippage or damages, and thus the IFSS value of the CF-PMEA/epoxy composite decreased more significantly and from $40.15 \pm 1.4$ to $38.3 \pm 1.5 \mathrm{MPa}$ and $36.0 \pm 1.4 \mathrm{MPa}$.

\subsubsection{Crosslinking by chemical bonds}

In this section, the carbon fibers grafted with diblock copolymers ( $\mathrm{PnBA}_{160}-c o$-PMEA 160 )- $b$-PGMA were subjected to UV light for irradiation before and after being incorporated with the epoxy resin, respectively. The fibers that were first incorporated with epoxy resin and cured, then subjected to UV irradiation, are denoted as curing-crosslinking samples; while those with the treatment steps contrary to the above ones are named as crosslinking-curing samples. During the UV irradiation, the PMEA moieties in the grafted copolymers reacted with each other, as discussed in section 3.3, resulting in the crosslinking of flexible layer by chemical bonds. The IFSS values of the CF-PMEA/epoxy resin composites after UV irradiation for different time (15 and $20 \mathrm{~min}$ ) through the two processes, respectively, are presented in Figure 12.

For the curing-crosslinking samples, the random copolymers PnBA-co-PMEA assembled into many hemispheres to form a perfect interfacial flexible layer on carbon fiber surface due to an entropic 


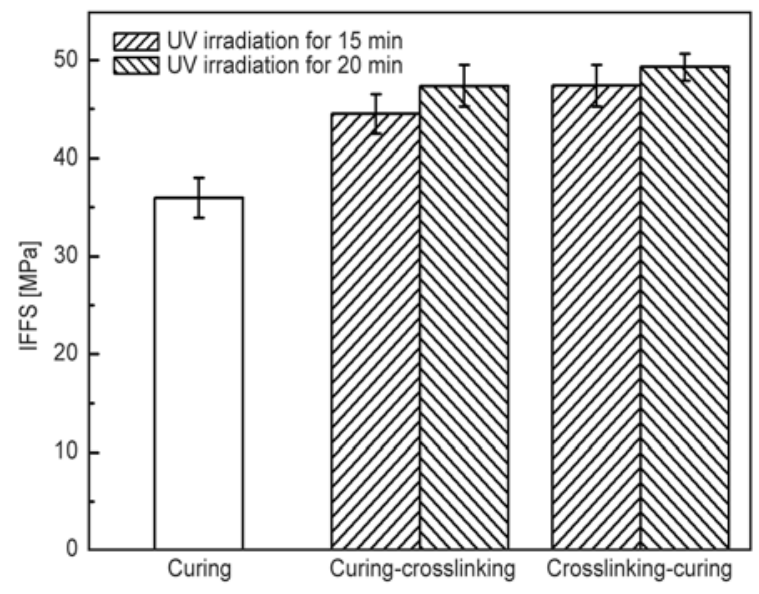

Figure 12. Effect of photo-crosslinking on IFSS of carbon fibers grafted with diblock copolymers $\left(\mathrm{PnBA}_{160^{-}}\right.$ co-PMEA 160 )- $b$-PGMA/epoxy resin composite

repulsion during the curing process of the composite (see Figure 13) [10]. In this case, the unreacted low-molecular-weight epoxy resin and curing agent would be very hard to enter into the assembled flexible layer during the following process. Therefore, such an interfacial assembled structure that can release stress and uniformly transfer load, was perfectly retained after UV irradiation, resulting in the obviously increased interfacial shear strength, from $36.0 \pm 2.1$ to $44.5 \pm 2.0 \mathrm{MPa}$ for $15 \mathrm{~min}$ and 47.4 $\pm 2.1 \mathrm{MPa}$ for $20 \mathrm{~min}$. However, since the molecular movement of the assembled hemispheres was difficult, the photo-crosslinking reactions between the PMEA moieties may tend to occur inside the isolated hemispheres. Thereby, the crosslinking between the hemispheres is less than the crosslinking inside the hemispheres, resulting in the crosslinked flexible interlayer with a regularly latticed distribution of crosslinking degree. Such an interfa-

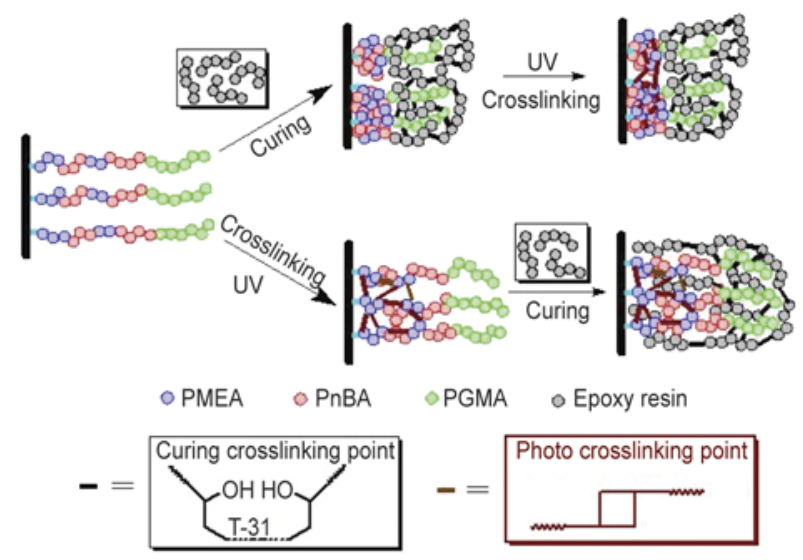

Figure 13. Illustration of the formation of the interfacial network structure crosslinking by chemical bonds cial crosslinked structure can preferably retain the whole deformation ability of interface, but may lead to the local over-crosslinking inside the hemispheres as the crosslinking reaction proceeding. When the carbon fibers were first irradiated with UV light, the PMEA moieties in the grafted copolymers reacted with each other, forming the crosslinking networks in the flexible layer, which could be very difficult to reassemble in the following compounding process with epoxy resin. In this case, the grafted diblock copolymers, existing in a single chain, had strong motion ability before UV irradiation, beneficial to the crosslinking reaction and consequently the improvement of the crosslinking degree of the flexible blocks. Furthermore, due to the low molecular weight of the unreacted epoxy resin, some of the matrix polymer chains and the curing agent could diffuse into the photo-crosslinking network in the curing process. Therefore, the interfacial photo-crosslinked network and the epoxy cured network would form an interpenetrating crosslinked network structure in the interface region as illustrated in Figure 13. Such an interfacial network structure possesses high strength and modulus, which will benefit for the load transfer at the interface. Therefore, the interfacial adhesion between carbon fiber and epoxy resin was strongly enhanced, corresponding to the results (as seen in Figure 12) that the IFSS value sharply increased to $47.4 \pm 2.1 \mathrm{MPa}$ after UV irradiation for $15 \mathrm{~min}$, and $49.3 \pm 1.4 \mathrm{MPa}$ for $20 \mathrm{~min}$.

However, it should be noted that for the CF-PMEA/ epoxy composite with the crosslinking-curing process, because of the formation of the interpenetrating crosslinked network and especially the small deformation ability of the epoxy crosslinked network, the whole deformation of the interfacial layer could be dramatically reduced, and thus the interfacial toughness of the composite may be decreased accompanying with the improvement of the interfacial adhesion.

\section{Conclusions}

In this study, a series of diblock copolymers (PnBA$c o$-PHEA)- $b$-PGMA containing one flexible random copolymer block PnBA-co-PHEA were successfully synthesized by ATRP. On one hand, the hydroxyl groups of the PHEA moieties in the flexible blocks can react with the functional groups on carbon fiber surfaces. On the other hand, the photo- 
crosslinkable MA groups can be introduced into the flexible blocks by reacting with the hydroxyl groups. Due to the existence of the hydroxyl groups in the PHEA moieties, an interfacial network structure crosslinking by H-bonds was formed. While with the introduction of the photo-crosslinkable MA groups into the interface, an interfacial network structure crosslinking by chemical bonds was finally received by UV irradiation before (crosslinking-curing) and after (curing-crosslinking) $\mathrm{CF}$ incorporated with epoxy resin.

Microbond test showed that the formation of the interfacial network structure via photo-crosslinking greatly improved the cohesive strength of the flexible blocks, corresponding to the increased $T_{\mathrm{g}}$, and thus effectively retained the whole deformation ability of the flexible interlayer. Furthermore, due to the formation of the interpenetrating crosslinked network structure between the photo-crosslinking network and the epoxy crosslinking network in the crosslinking-curing process, which possesses high strength and modulus and thus benefit for the load transfers of the interface, the interfacial properties of the CF/epoxy composites cured by the curingcrosslinking process and by crosslinking-curing process were slightly different. Consequently, the interfacial shear strength increased from $36.0 \pm 2.1$ to $40.5 \pm 2.5 \mathrm{MPa}$ for the interfacial network structure crosslinking by H-bonds, $47.4 \pm 2.1 \mathrm{MPa}$ for 20 min UV irradiation in the curing-crosslinking process, and $49.3 \pm 1.4 \mathrm{MPa}$ in the crosslinking-curing process.

\section{Acknowledgements}

The authors sincerely acknowledge the support of NSFC 51073055 and the Fundamental Research Funds for the Central Universities.

\section{References}

[1] Pearson R. A., Yee A. F.: Toughening mechanisms in elastomer-modified epoxies. Journal of Materials Science, 24, 2571-2580 (1989).

DOI: $10.1007 / \mathrm{BF} 01174528$

[2] Kuwata M., Hogg P. J.: Interlaminar toughness of interleaved CFRP using non-woven veils: Part 1. Mode-I testing. Composites Part A: Applied Science and Manufacturing, 42, 1551-1559 (2011).

DOI: $10.1016 /$ j.compositesa.2011.07.016
[3] Wong D. W. Y., Lin L., MaGrail P. T., Peijs T., Hogg P. J.: Improved fracture toughness of carbon fibre/epoxy composite laminates using dissolvable thermoplastic fibres. Composites Part A: Applied Science and Manufacturing, 41, 759-767 (2010).

DOI: $10.1016 /$ j.compositesa.2010.02.008

[4] Gkikas G., Barkoula N-M., Paipetis A. S.: Effect of dispersion conditions on the thermo-mechanical and toughness properties of multi walled carbon nanotubes-reinforced epoxy. Composites Part B: Engineering, 43, 2697-2705 (2012).

DOI: $10.1016 /$ j.compositesb.2012.01.070

[5] Jiang Z. X., Liu L., Huang Y. D., Ren H.: Influence of coupling agent chain lengths on interfacial performances of carbon fiber and polyarylacetylene resin composites. Surface and Interface Analysis, 41, 624-631 (2009).

DOI: 10.1002/sia.3074

[6] Tsubokawa N., Abe N., Wei G., Chen J., Saitoh S., Fujiki K.: Grafting of polymers onto a carbon-fiber surface by ligand-exchange reaction of poly(vinyl ferrocene-co-vinyl monomer) with polycondensed aromatic rings of the surface. Journal of Polymer Science Part A: Polymer Chemistry, 40, 1868-1875 (2002).

DOI: $10.1002 /$ pola.10268

[7] Severini F., Formaro L., Pegoraro M., Posca L.: Chemical modification of carbon fiber surfaces. Carbon, 40, 735-741 (2002).

DOI: $10.1016 / \mathrm{S} 0008-6223(01) 00180-4$

[8] Gerard J-F.: Characterization and role of an elastomeric interphase on carbon fibers reinforcing an epoxy matrix. Polymer Engineering and Science, 28, 568-577 (1988).

DOI: $10.1002 /$ pen.760280905

[9] Ishida I., Chaisuwan T.: Mechanical property improvement of carbon fiber reinforced polybenzoxazine by rubber interlayer. Polymer Composites, 24, 597-607 (2003).

DOI: $10.1002 / p c .10056$

[10] Li Y., Lin Q., Chen L., Zhou X.: Assembly of triblock copolymer brush at glass fiber/polystyrene interface and its effect on interfacial shear strength. Composites Science and Technology, 69, 1919-1924 (2009). DOI: 10.1016/j.compscitech.2009.04.008

[11] Shen T., Zhou X., Lin Q.: Influence of grafting density of diblock copolymers on interfacial assembly behavior and interfacial shear strength of glass fiber/polystyrene composites. Composite Interfaces, 18, 701714 (2011). DOI: $10.1163 / 156855412 X 626270$

[12] Zhou X., Ying S.: Basalt fibres/polystyrene interfacial adhesion through modification of basalt fibres by block copolymers. Iranian Polymer Journal, 20, 571578 (2011). 
[13] Deng S., Zhou X., Fan C., Lin Q., Zhou X.: Release of interfacial thermal stress and accompanying improvement of interfacial adhesion in carbon fiber reinforced epoxy resin composites: Induced by diblock copolymers. Composites Part A: Applied Science and Manufacturing, 43, 990-996 (2012).

DOI: $10.1016 /$ j.compositesa.2012.01.004

[14] Pilon L. N., Armes S. P., Findlay P., Rannard S. P.: Synthesis and characterisation of new shell cross-linked micelles with amine-functional coronas. European Polymer Journal, 42, 1487-1498 (2006).

DOI: 10.1016/j.eurpolymj.2006.01.006

[15] Tillet G., Boutevin B., Ameduri B.: Chemical reactions of polymer crosslinking and post-crosslinking at room and medium temperature. Progress in Polymer Science, 36, 191-217 (2011). DOI: 10.1016/j.progpolymsci.2010.08.003

[16] Zheng Y., Andreopoulos F. M., Micic M., Huo Q., Pham S. M., Leblanc R. M.: A novel photoscissile poly (ethylene glycol)-based hydrogel. Advanced Functional Materials, 11, 37-40 (2001).

DOI: $10.1002 / 1616-3028(200102) 11: 1<37:: A I D-$ ADFM37>3.0.CO;2-V

[17] Kadam V. S., Nicol E., Gaillard C.: Synthesis of flower-like poly(ethylene oxide) based macromolecular architectures by photo-cross-linking of block copolymers self-assemblies. Macromolecules, 45, 410-419 (2012).

DOI: $10.1021 / \mathrm{ma} 2022937$

[18] Kim J. S., Youk J. H.: Preparation of core cross-linked micelles using a photo-cross-linking agent. Polymer, 50, 2204-2208 (2009).

DOI: $10.1016 /$ j.polymer.2009.03.013

[19] Sonoda Y.: Solid-state [2+2] photodimerization and photopolymerization of $\alpha, \omega$-diarylpolyene monomers: Effective utilization of noncovalent intermolecular interactions in crystals. Molecules, 16, 119-148 (2011). DOI: 10.3390/molecules16010119

[20] Stauch O., Uhlmann T., Fröhlich M., Thomann R., ElBadry M., Kim Y-K., Schubert R.: Mimicking a cytoskeleton by coupling poly( $N$-isopropylacrylamide) to the inner leaflet of liposomal membranes: Effects of photopolymerization on vesicle shape and polymer architecture. Biomacromolecules, 3, 324-332 (2002). DOI: $10.1021 / \mathrm{bm} 015613 \mathrm{y}$

[21] Piogé S., Nesterenko A., Brotons G., Pascual S., Fontaine L., Gaillard C., Nicol E.: Core cross-linking of dynamic diblock copolymer micelles: Quantitative study of photopolymerization efficiency and micelle structure. Macromolecules, 44, 594-603 (2011). DOI: $10.1021 / \mathrm{ma102284y}$
[22] Hou J., Zhou X., Zhou X.: Grafting of poly(n-butylacrylate)- $b$-poly(2-hydroxyethyl methacrylate) on carbon fiber and its effect on composite properties. Polymer-Plastics Technology and Engineering, 50, 260265 (2011). DOI: $10.1080 / 03602559.2010 .531863$

[23] He J. M., Huang Y. D.: Effect of silane-coupling agents on interfacial properties of CF/PI composites. Journal of Applied Polymer Science, 106, 2231-2237 (2007). DOI: $10.1002 / \mathrm{app} .26875$

[24] Deng S., Ye H., Jiang D., Fan C., Lin Q., Zhao J., Zhou $\mathrm{X}$.: Copolymerization and reactivity ratio measurement of n-butylacrylate and hydroxyethyl acrylate (in Chinese). Engineering Plastics Application, 41, 92-95 (2013). DOI: 10.3969/J.ISSN.1001-3539.2013.09.020

[25] Jin L., Agag T., Yagci Y., Ishida H.: Methacryloylfunctional benzoxazine: Photopolymerization and thermally activated polymerization. Macromolecules, 44, 767-772 (2011). DOI: $10.1021 / \mathrm{ma102351 \textrm {a }}$

[26] Pedrón S., Bosch P., Peinado C.: Using hyperbranched macromers as crosslinkers of methacrylic networks prepared by photopolymerization. Journal of Photochemistry and Photobiology A: Chemistry, 200, 126140 (2008). DOI: $10.1016 /$ j.jphotochem.2008.07.002

[27] Maruyama K., Kudo H., Ikehara T., Ito N., Nishikubo T.: Synthesis of photocrosslinkable hyperbranched polyesters and their film properties. Journal of Polymer Science Part A: Polymer Chemistry, 43, 46424653 (2005). DOI: $10.1002 /$ pola.20957

[28] Maruyama K., Kudo H., Ikehara T., Nishikubo T.: Synthesis and properties of photo-cross-linkable hyperbranched poly(urethane)s containing both terminal methacryloyl groups and carboxyl groups. Macromolecules, 40, 4895-4900 (2007).

DOI: $10.1021 / \mathrm{ma} 062381 \mathrm{~s}$

[29] Chartoff R. P., Menczel J. D., Dillman S. H.: Dynamic mechanical analysis (DMA). in 'Thermal analysis of polymers: Fundamentals and applications' (eds.: Menczel J. D., Prime R. B.). Wiley, New York, 438439 (2009).

[30] Lewin J. L., Maerzke K. A., Schultz N. E., Ross R. B., Siepmann J. I.: Prediction of hildebrand solubility parameters of acrylate and methacrylate monomers and their mixtures by molecular simulation. Journal of Applied Polymer Science, 116, 1-9 (2010). DOI: 10.1002/app.31232 